\title{
Effects of Staphylococcus aureus intramammary infection on the expression of estrogen receptor $\alpha$ and progesterone receptor in mammary glands of nonlactating cows administered estradiol and progesterone to stimulate mammary growth
}

\author{
B. D. Enger, ${ }^{1}$ H. L. M. Tucker, ${ }^{1}$ S. C. Nickerson, ${ }^{2}$ C. L. M. Parsons, ${ }^{1}$ and R. M. Akers ${ }^{1 *}$ \\ ${ }^{1}$ Dairy Science Department, Virginia Polytechnic Institute and State University, Blacksburg 24060 \\ ${ }^{2}$ Animal and Dairy Science Department, University of Georgia, Athens 30602
}

\section{ABSTRACT}

Intramammary infections (IMI) are prevalent in nonlactating dairy cattle and are known to alter mammary structure and negatively affect the amount of mammary epithelium in the gland. Mechanisms responsible for the observed changes in mammary growth during an IMI are poorly understood, yet the importance of the key mammogenic hormones driving mammary growth is well recognized. This study's objective was to characterize the expression of estrogen receptor $\alpha$ (ESR1) and progesterone receptor (PGR) in mammary glands stimulated to grow and develop in the presence or absence of an IMI as well as preliminarily characterize myoepithelial cell response to IMI. Mammary growth was stimulated in 18 nonpregnant, nonlactating dairy cows using subcutaneous estradiol and progesterone injections, and 2 culture-negative quarters of each cow were subsequently infused with either saline $(\mathrm{n}=18)$ or Staphylococcus aureus $(\mathrm{n}=18)$. Mammary parenchyma tissues were collected $5 \mathrm{~d}(\mathrm{n}=9)$ or 10 $\mathrm{d}(\mathrm{n}=9)$ postchallenge and examined using immunofluorescence microscopy to quantify positive nuclei and characterize staining features. There tended to be a greater number of ESR1-positive nuclei observed across 8 random mammary parenchyma fields of view in saline quarters than in Staph. aureus quarters (201 vs. 163 \pm 44 nuclei). Saline quarters also contained a greater number of PGR-positive nuclei (520 vs. $440 \pm 45 \mathrm{nu}$ clei) and myoepithelial cells (971 vs. $863 \pm 48$ nuclei) than Staph. aureus-challenged quarters. However, when ESR1, PGR, and myoepithelial nuclei counts were adjusted for Staph. aureus quarters containing less epithelium, differences between quarter treatments abated. The examined ESR1 and PGR staining characteristics

Received August 2, 2018.

Accepted November 20, 2018.

*Corresponding author: rma@vt.edu were similar between saline and Staph. aureus quarters but were differentially affected by day of tissue collection. Additionally, nuclear staining area of myoepithelial cells was greater in Staph. aureus quarters than in saline quarters. These results indicate that IMI had little effect on the number or staining characteristics of ESR1- or PGR-positive nuclei relative to epithelial area, but myoepithelial cells appear to be affected by IMI and the associated inflammation in nonlactating mammary glands that were stimulated to grow rapidly using mammogenic hormones. Accordingly, reductions in mammary epithelium in affected glands are not suspected to be resultant of alterations in the number or staining characteristics of ESR1- or PGR-positive mammary epithelial cells.

Key words: mastitis, dry cow, heifer, mammary growth, myoepithelial cell

\section{INTRODUCTION}

A primary goal in the rearing and management of production dairy animals is to maximize mammary gland growth and development. This goal is emphasized because milk yield is solely influenced by the number of secretory mammary epithelial cells in the gland and their activity; any improvements or impairments in either of these 2 factors would ultimately influence milk yields.

It is not by chance that considerable amounts of mammary growth and development occur during gestation. The maturation and growth of the mammary gland during gestation are primarily driven by the pregnancy-associated hormones estradiol and progesterone and are essential to allow for the development of a gland that is capable of synthesizing and secreting milk to support the neonate. Before first gestation, limited amounts of mammary parenchyma are present, and no functional alveolar structures exist that would synthesize and secrete these milk components. To align 
with the need for the development and maturation of the gland, substantial mammary growth occurs during first pregnancy, which is when the greatest amount of mammary growth and development occurs during an animal's life (Tucker, 1987).

Appreciable amounts of mammary growth also occur in dry cows during the second half of the dry period that coincides with late pregnancy, which contributes to the increased milk yields realized in succeeding lactations (Capuco et al., 1997; Capuco and Akers, 1999). Given the considerable amounts of mammary growth that occur during the distinct physiological states of gestation, it is understandable that impairments in mammary growth during this time would have negative effects on future milk yields.

The chief mechanisms driving mammary growth and morphogenesis have been reviewed previously (Lyons et al., 1958; Knight and Peaker, 1982; Akers, 1990, 2017; Tucker, 2000), but it is irrefutably recognized that estradiol and progesterone play central roles in stimulating mammary epithelial cell proliferation (Woodward et al., 1993; Capuco et al., 2002) and promote lobular-alveolar development and glandular morphogenesis (Sud et al., 1968; Howe et al., 1975; Croom et al., 1976). The actions that estradiol and progesterone have in promoting mammary gland growth are largely dependent on these steroid hormones binding with their nuclear receptors, estrogen receptor $\alpha$ (ESR1) and progesterone receptor (PGR), in mammary epithelial cells to induce production of mitogenic factors that promote mammary growth and development (Connor et al., 2007). Changes in the expression of these receptors in the mammary epithelium has been associated with changes in mammary growth (Meyer et al., 2006; Tucker et al., 2016b).

Coincident with these periods of rapid mammary growth during pregnancy is the occurrence of bacterial IMI, which are common in primigravid dairy heifers (Trinidad et al., 1990b; Fox, 2009) and dry cows (Eberhart, 1986). For instance, heifer IMI prevalence at the quarter level is approximately $43 \%$ (Fox, 2009), whereas 8 to $25 \%$ of dry cow quarters become newly infected between lactations (Arruda et al., 2013). Intramammary infections in the mammary glands of nonpregnant heifers (Trinidad et al., 1990a) as well as nonpregnant dry cows (Andreotti et al., 2014) have been shown to induce leukocyte migration, increase stromal tissue areas, and reduce mammary epithelial tissue present in infected glands, demonstrating that IMI negatively affect gland structure in nonlactating glands. However, in these instances, limited amounts of mammary growth and development are expected given that these animals are not pregnant. Yet, previous investigations have reported that infections during first gestation, when considerable amounts of mammary growth are expected, reduce milk yields by approximately $11 \%$ (Owens et al., 1991; Oliver et al., 2003). When these observations are evaluated, it indicates that IMI during periods of rapid mammary growth can negatively affect the amount of epithelium in the gland, which is expected to reduce future milk yields.

Recently, Enger et al. (2018) stimulated rapid mammary growth and development in glands from nonpregnant dry cows via controlled injections of estradiol and progesterone at supraphysiological levels and subsequently challenged these glands with Staphylococcus aureus to investigate how IMI, and the resulting immune response, affected the mammary gland. As expected, leukocytosis, increased areas of stromal tissue, and reductions in mammary epithelium resulted; however, it is unknown whether IMI reductions in mammary epithelium are influenced by estradiol and progesterone signaling. In this report, we studied these previously collected tissues using immunohistochemistry and quantitative image analysis approaches to investigate whether Staph. aureus IMI affected expression of ESR1 and PGR in bovine mammary glands that were stimulated to rapidly grow and develop. Additionally, a preliminary evaluation of myoepithelial epithelial cells in these tissues was conducted.

\section{MATERIALS AND METHODS}

\section{Study Design}

The mammary tissues used in this study were collected in a previous study by Enger et al. (2018). In brief, 19 nonpregnant, nonlactating dairy cows that had been dry for $45 \mathrm{~d}$ were administered daily subcutaneous injections of estradiol $(0.1 \mathrm{mg} / \mathrm{kg}$ of BW; Sigma-Aldrich, St. Louis, MO; cat. no. E1024) and progesterone (0.25 $\mathrm{mg} / \mathrm{kg}$ of BW; Sigma-Aldrich; cat. no. P3972) for $1 \mathrm{wk}$ to stimulate mammary growth and development. We have used this hormonal induction regimen previously with these same dosages (Nickerson et al., 1978; Ball et al., 2000). Three days after the last injection, 2 culturenegative quarters of each cow were infused with either sterile phosphate-buffered physiological saline $(\mathrm{n}=19)$ or Staph. aureus $(\mathrm{n}=19)$. All saline-infused quarters remained culture negative throughout the trial, and all but 1 cow developed a Staph. aureus IMI in the challenged quarters that persisted until tissue collection, as determined by secretion culture. At 5 d postchallenge, half of the cows were randomly selected and euthanized for tissue collection; tissues were collected in the same manner from the remaining animals at $10 \mathrm{~d}$ postchallenge. Mammary parenchyma proximal to the teat but dorsal to the gland cistern was collected from experimental quarters and fixed in formalin before being 

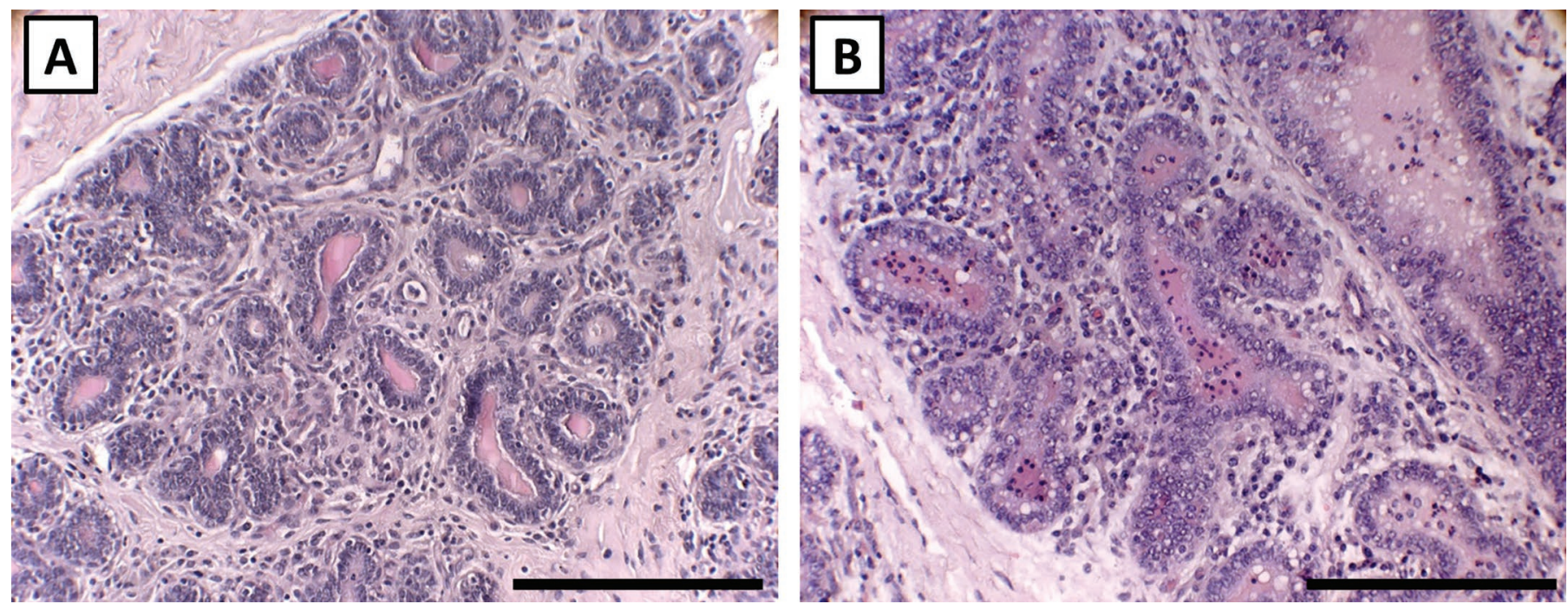

Figure 1. Example hematoxylin and eosin images of mammary tissues collected from a saline-infused quarter (A) and a Staphylococcus aureus-infused quarter (B). Enger et al. (2018) previously observed that neutrophils were seldom present in mammary tissues from saline-infused quarters, whereas neutrophils were common and abundant in both the luminal and interlobular stroma compartments of Staph. aureus-infused quarter tissues. Lymphocytes were present in interlobular stroma compartment of saline-infused quarter mammary tissues but were more abundant in Staph. aureus-infused quarter tissues while also being abundant in the luminal spaces. Enger et al. (2018) also previously reported that Staph. aureus-infused quarters had less epithelial area and a greater amount of luminal space and tended to have more interlobular stroma than saline-infused quarters. Scale bars $=200 \mu \mathrm{m}$.

embedded in paraffin. The mammary tissues collected from the animal that did not establish a Staph. aureus IMI were not used in the present investigation. Images of hematoxylin and eosin stained mammary tissues from both saline- and Staph. aureus-infused quarters are presented in Figure 1.

\section{Immunohistochemistry}

Two slide sets were prepared to examine ESR1 and PGR staining in mammary epithelial cells from saline and Staph. aureus-infused mammary quarters. Myoepithelial cells were also identified in both the ESR1 and PGR slide sets via p40 staining (Parsons et al., 2018) to act as a morphological marker, and the myoepithelial cells identified in the PGR slide set were subject to examination. Tissues were sectioned $5 \mu \mathrm{m}$ thick using a rotary microtome (model HM 340 E, Microm International GmbH, Waldoff, Germany) and placed in a $42^{\circ} \mathrm{C}$ water bath. For each slide set, 2 serial sections from each experimental quarter were mounted on Superfrost Plus microscope slides (Thermo Fisher Scientific, Waltham, MA), drained, and dried at $37^{\circ} \mathrm{C}$ for $24 \mathrm{~h}$. Sections were deparaffinized in 3 changes of a xylene substitute (Clear-Rite 3, Thermo Fisher Scientific) and rehydrated to deionized water using a graded ethanol series. Antigens were retrieved by immersing slides in $10 \mathrm{~m} M$ citrate buffer, $\mathrm{pH} 6.0$, for $30 \mathrm{~min}$ at $95^{\circ} \mathrm{C}$. Slides were removed from the heat source after antigen re- trieval and allowed to cool to room temperature while remaining submerged in citrate buffer. Slides were subsequently washed in $0.9 \% \mathrm{NaCl}$ PBS, $\mathrm{pH} 7.4$, and a hydrophobic barrier pen (Liquid Blocker, Daido Sangyo Co. Ltd., Saitama, Japan) was used to separate tissue sections on the same slide to prevent antibody mixtures on different sections from comingling. All sections were subsequently blocked using a universal blocking agent (CAS Block, Thermo Fisher Scientific) for 30 min.

The CAS Block was aspirated from the sections, and $100 \mu \mathrm{L}$ of a primary antibody mixture was applied to a single section on each slide; the remaining section served as the negative control and was covered with $100 \mu \mathrm{L}$ of CAS Block. The primary antibody mixture used for the ESR1 slide set included an antibody to detect ESR1 and an antibody to detect p40 (Table 1). The primary antibody mixture used for the PGR slide set included an antibody to detect PGR and the p40 antibody (Table 1). Antibodies were mixed in CAS Block using the dilutions specified in Table 1 and incubated with the respective slide sets in a dark humidified chamber at $4^{\circ} \mathrm{C}$ for $16 \mathrm{~h}$. After primary antibody incubation, solutions were aspirated, and sections were washed thrice with PBS for 5 min each washing. All tissue sections were then incubated with $100 \mu \mathrm{L}$ of a secondary antibody mixture at room temperature for $60 \mathrm{~min}$ in a dark humidified chamber to detect primary antibodies with the desired fluorophore. Secondary antibodies were also suspended in CAS Block at the dilutions specified in 
Table 1. Antibodies used to stain estrogen receptor $\alpha$ (ESR1)-, progesterone receptor (PGR)-, and p40-positive nuclei in mammary tissues

\begin{tabular}{|c|c|c|c|c|c|}
\hline Antibody and type & Manufacturer $^{1}$ & Host and isotype & $\begin{array}{l}\text { Target } \\
\text { antigen }\end{array}$ & $\begin{array}{l}\text { Catalog } \\
\text { no. }\end{array}$ & Dilution \\
\hline $\mathrm{ERS} 1,1^{\circ}$ & Santa Cruz Biotechnology & Rabbit IgG, polyclonal & ESR1 & SC-787 & $1: 100$ \\
\hline $\mathrm{PGR}, 1^{\circ}$ & Santa Cruz Biotechnology & Mouse $\mathrm{IgG}_{2 \mathrm{a}}$, monoclonal & PGR & SC-7208 & $1: 200$ \\
\hline Alexa Fluor $594,2^{\circ}$ & Thermo Fisher Scientific & Goat IgG, polyclonal & Mouse $\operatorname{IgG}_{2 \mathrm{a}}$ & A-21135 & $1: 200$ \\
\hline Alexa Fluor $594,2^{\circ}$ & Thermo Fisher Scientific & Goat IgG, polyclonal & Rabbit IgG & A-11037 & $1: 200$ \\
\hline Alexa Fluor $488,2^{\circ}$ & Thermo Fisher Scientific & Goat IgG, polyclonal & Mouse $\operatorname{IgG}_{1}$ & A-21121 & $1: 200$ \\
\hline
\end{tabular}

${ }^{1}$ Santa Cruz Biotechnology, Dallas, TX; Abchem, Elroy, WI; Thermo Fisher Scientific, Waltham, MA.

Table 1. Secondary antibody mixtures were aspirated and sections were washed thrice more with PBS for 2 min each washing. Residual hydrophobic barrier pen outlines were removed and sections were coverslipped using ProLong Gold Antifade Mountant containing 4',6-diamidino-2-phenylindole (DAPI; Thermo Fisher Scientific). The coverslipping mountant was cured overnight before sections were imaged.

\section{Imaging and Image Analysis}

Eight mammary parenchyma fields of view were randomly identified and imaged for each experimental quarter tissue section. Regions of mammary parenchyma were identified using DAPI nuclear staining as a morphological index. Images were acquired using a Nuance FX multispectral imaging system (Perkin Elmer, Waltham, MA) affixed to a Nikon Eclipse E600 epifluorescence microscope (Nikon Instruments Inc., Melville, NY) with a Plan Fluor $40 \times$ objective. A mercury lamp was used as the excitation light source, and filter cubes, fitted with long pass emission filters, were used to excite specific secondary antibody and DAPI fluorophores. The Nuance system was configured to use multiple tailored emission spectra to visualize DAPI-counterstained nuclei and positively stained ESR1, PGR, and p40 nuclei. As a result, 3 images for each field of view were produced for each slide set and used for subsequent analysis: 1 image depicted DAPIcounterstained nuclei, 1 depicted p40-stained nuclei, and 1 depicted either ESR1- or PGR-positive nuclei depending on the slide set. Correspondingly, 864 images were produced from the 288 imaged fields of view for each slide set.

Analysis of the acquired images was 2-fold and included an enumeration of the number of positively stained nuclei and characterization of positively stained nuclei. For each respective slide set, the number of positively stained ESR1 or PGR nuclei in each field of view was manually counted using a composite image (DAPI + p40 + ERS1 or PGR), and the number of positively stained p40 nuclei identified in the PGR slide set was enumerated by CellProfiler 2.2.0 (Kamentsky et al., 2011). Counts produced for each field of view were summed to produce a total number of positive cells across the 8 imaged fields of view of mammary parenchyma, similar to the approach described previously (Long et al., 2001). With the microscope configuration used, the total tissue area analyzed in 8 microscopic fields was $0.718 \mathrm{~mm}^{2}$.

CellProfiler 2.2.0 (Kamentsky et al., 2011) was used to analyze morphological and staining characteristics of cell nuclei that were positively stained for ESR1, PGR, and $\mathrm{p} 40$. This was achieved by creating 2 CellProfiler pipelines that were unique to the ESR1 or PGR slide sets. In each pipeline, the 3 images for each field of view were submitted and designated as being from the same field of view. First, all nuclei in the DAPI-counterstained image were identified and defined as areas of interest that could be examined further in companion images of the same field of view to identify positively stained nuclei. This approach was used to eliminate background signal (cytoplasmic and stromal autofluorescence or secondary antibody aggregates) that could be incorrectly classified as positively stained nuclei. Objects identified as positively stained ESR1, PGR, and p40 nuclei were outlined by CellProfiler on the entered images, and the resulting images were inspected to confirm that positive nuclei were correctly identified as a quality control measure. In instances where objects were not correctly identified, artifacts were manually effaced, and the image was reanalyzed by CellProfiler. If nuclei were still not correctly identified, the image was removed.

For the ESR1 slide set pipeline, CellProfiler intensity measures (mean intensity and integrated intensity) and area and shape measures [object size (area) and object eccentricity (roundness)] were applied to all ESR1identified nuclei objects. These same measures were applied to all PGR-positive nuclei objects identified in the PGR slide set. Positively stained p40 nuclei were examined in the PGR slide set only and subject to area and shape measures (object size and object eccentricity). An overall mean for the respective measures was calculated for each experimental quarter using the objects identified across all the analyzed fields of view. 


\section{Statistical Analysis}

The total number of positively stained ESR1 and PGR mammary epithelial cells quantified from the 8 images per experimental quarter were analyzed using the MIXED procedure in SAS 9.4 (SAS Institute Inc., Cary, NC). Total positive ESR1 and PGR cell counts served as the dependent variable in separate models. The fixed independent effects of quarter treatment (n $=2)$ and euthanasia day $(\mathrm{n}=2)$ were included in both models; their interaction was included when $P \leq 0.10$. Cow nested within day of euthanasia was specified as a random effect in both models. For the ESR1 data set, positive ESR1 nuclei counts were non-normally distributed and produced non-normally distributed residuals when tested against the model's effects. In response, $\log _{10}$-transformed counts of ESR1-positive nuclei were computed and analyzed to satisfy the assumptions of normal distribution and equal variance. Least squares means were estimated using untransformed positive ESR1 and PGR nuclei counts, but $P$-values produced by the $\log _{10}$-transformed ERS1 model are reported. Least squares means produced by the models were contrasted using Fisher's least significant difference test.

CellProfiler intensity, area, and eccentricity measures for ESR1-, PGR-, and p40-stained nuclei were analyzed using the MIXED procedure as dependent variables in separate models. The fixed independent effects of quarter treatment $(\mathrm{n}=2)$ and euthanasia day $(\mathrm{n}=2)$ were included in each model; their interaction was included if $P \leq 0.10$. Cow nested within day of euthanasia was specified as random effect in all models. Resultant least squares means estimated by the models were contrasted using Fisher's least significant difference test.

\section{RESULTS}

\section{ESR1-Positive Nuclei Counts and Staining Characteristics}

Representative images of ESR1 staining are presented in Figure 2, and the sums of ESR1-positive nuclei least squares means counts are summarized and contrasted in Table 2. Total ESR1-positive nuclei counts in saline-infused quarters tended to be higher than those in Staph. aureus-infused quarters (201 vs. $163 \pm 44$ nuclei; $P=0.098)$. Additionally, ESR1-positive nuclei counts were lower in mammary tissues collected $5 \mathrm{~d}$ postinfusion than in tissues collected $10 \mathrm{~d}$ postinfusion (130 vs. $234 \pm 59$ nuclei; $P=0.044$ ). Positive ESR1 nuclei counts were not influenced by quarter treatment interacting with day of euthanasia $(P=0.51)$.

A total of 256 imaged fields of view were ultimately analyzed to examine ESR1-stained nuclei character- istics; 7,118 ESR1-stained nuclei were identified from these images and subject to the described CellProfiler measures. The least squares means produced for the selected staining characteristics of positively stained ESR1 nuclei are also presented and contrasted in Table 2 for the main effects of quarter treatment and day of euthanasia. In positively stained ESR1 nuclei, stained nuclear area was not found to differ between salineand Staph. aureus-infused quarters $(P=0.81)$, and positively stained nuclear area was not influenced by day of euthanasia $(P=0.56)$. Eccentricity (a unitless measure of roundness) of ESR1 nuclear staining was similar between positively stained nuclei in saline- and Staph. aureus-infused quarters $(P=0.32)$ and was not influenced by day of euthanasia $(P=0.51)$. Mean intensity of staining in ESR1-positive stained nuclei did not differ between saline- and Staph. aureus-infused quarters $(P=0.106)$, nor did mean integrated intensity of ESR1-positive nuclei $(P=0.30)$. Neither mean nor integrated intensities were influenced by day of euthanasia $(P \geq 0.28)$. No interaction was observed between day of euthanasia and quarter treatment on any of the ESR1 nuclear staining characteristics measured $(P \geq$ $0.24)$.

\section{PGR-Positive Nuclei Counts and Staining Characteristics}

Representative images of PGR staining are presented in Figure 2, and the summed PGR-positive nuclei least squares means counts are presented and contrasted in Table 3. Saline-infused quarters contained a greater number of summed PGR-positive nuclei counts than Staph. aureus-infused quarters (520 vs. $440 \pm 45 \mathrm{nu}-$ clei; $P=0.024)$, but positive PGR nuclei counts were not influenced by day of euthanasia $(P=0.78)$. No interaction was observed between the effects of day of euthanasia and quarter treatment on summed PGR counts $(P=0.93)$.

A total of 280 imaged fields of view were ultimately used to examine PGR-stained nuclei characteristics; 19,230 PGR-stained nuclei were identified from these images and subjected to the described CellProfiler measures. The least squares means staining characteristics for positively stained PGR nuclei are presented and contrasted in Table 3 for the main effects of quarter treatment and day of euthanasia. Nuclear staining area of positively stained PGR nuclei was not different between saline- and Staph. aureus-infused quarters $(P=$ 0.98), but the area of nuclear staining in PGR-stained nuclei was significantly lower in tissues collected $5 \mathrm{~d}$ postchallenge than in those collected $10 \mathrm{~d}$ postchallenge (477 vs. $545 \pm 19$ pixels; $P=0.022$ ). Eccentricity of 
PGR nuclear staining was not different between salineand Staph. aureus-infused quarters, but PGR staining in nuclei of tissues collected $5 \mathrm{~d}$ postchallenge (eccentricity $=0.672)$ tended $(P=0.056)$ to be less round than in tissues collected $10 \mathrm{~d}$ postchallenge (eccentricity $=0.652$ ). Mean intensity of PGR-stained nuclei was not different between saline- and Staph. aureus-infused quarters $(P=0.36)$ or in tissues collected 5 or $10 \mathrm{~d}$ post- challenge $(P=0.21)$. The mean integrated intensity of PGR-stained nuclei did not differ between saline- and Staph. aureus-infused quarters $(P=0.72)$; however, the integrated intensity of PGR-stained nuclei tended to be lower in tissues collected $5 \mathrm{~d}$ postchallenge than in tissues collected $10 \mathrm{~d}$ postchallenge (35.9 vs. $44.6 \pm 3.0 ; P$ $=0.055)$. No interaction was detected between quarter treatment and day of euthanasia in their effect on any

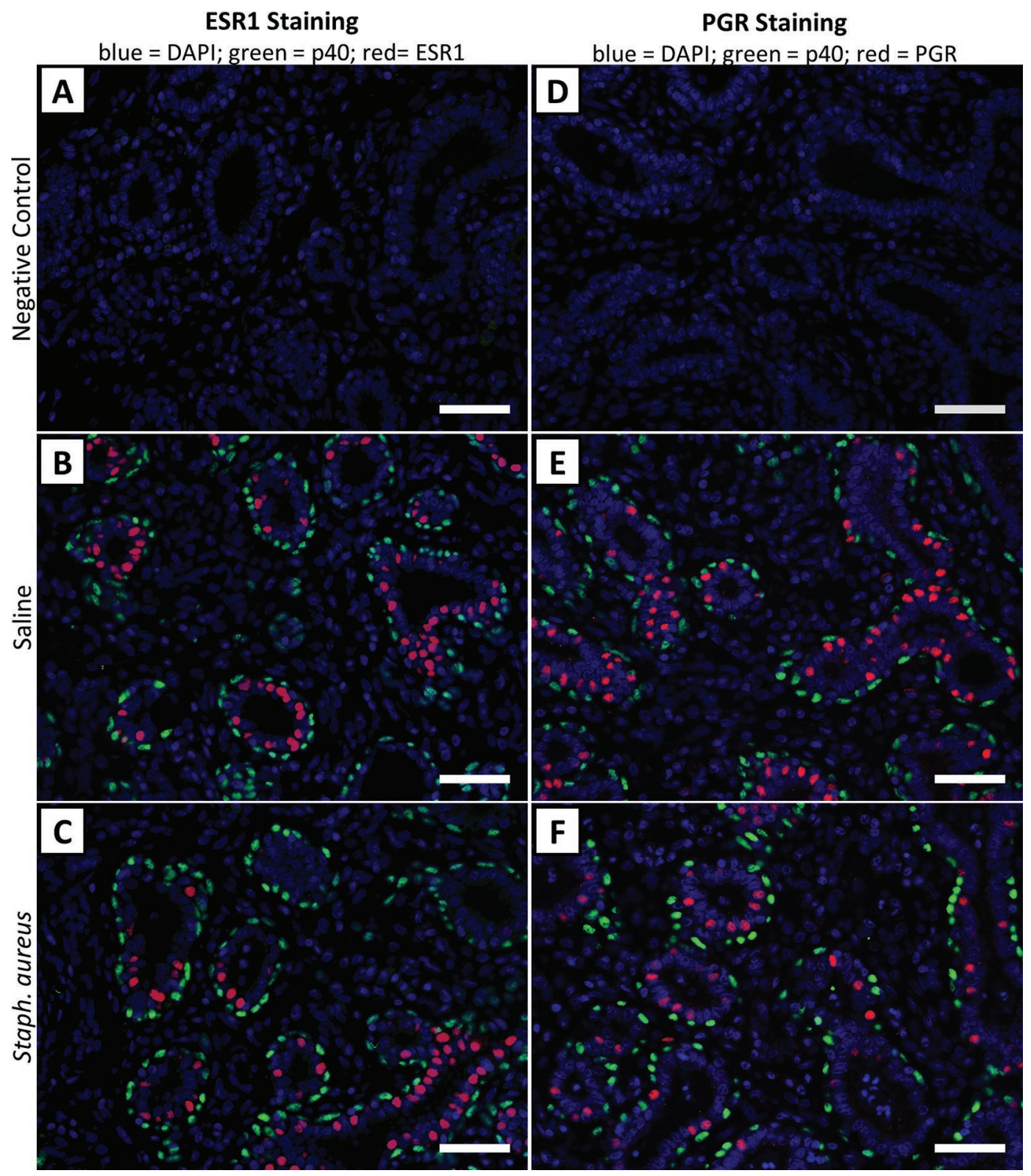

Figure 2. Staining of estrogen receptor $\alpha$ (ESR1; A-C), progesterone receptor (PGR; D-F), and p40 (a myoepithelial cell marker; B, C, E, and F) in saline- and Staphylococcus aureus-infused mammary gland tissues. Panels A and D depict negative control sections where the primary ESR1 or PGR and p40 antibodies were omitted; nuclei are counterstained with 4',6-diamidino-2-phenylindole (DAPI; blue). Panels B and E depict ESR1 and PGR (red) staining in tissues from saline-infused glands; myoepithelial cells are labeled by p40 staining (green). Panels C and F depict ESR1 and PGR staining in Staph. aureus tissues along with p40 staining. Scale bars $=50 \mu \mathrm{m}$. 
Table 2. Least squares means counts and staining characteristics of estrogen receptor $\alpha$ (ESR1)-positive nuclei in saline- and Staphylococcus aureus-infused mammary gland tissues

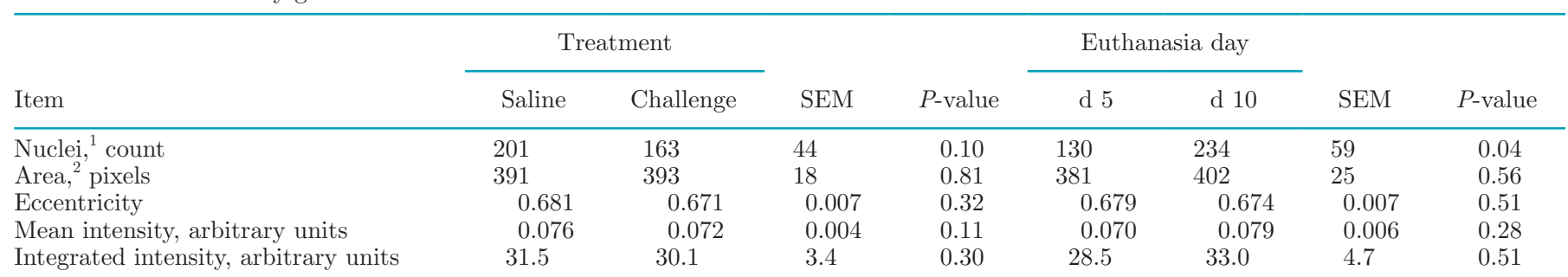

${ }^{1}$ Data expressed as the number of positive cells observed in 8 parenchymal tissue fields of view.

${ }^{2}$ Mean nuclear area of ESR1 staining in positively stained nuclei.

of the PGR nuclear staining characteristics measured $(P \geq 0.21)$.

\section{p40-Positive Nuclei Counts and Staining Characteristics}

All 288 imaged fields of view were used to quantify the number of p40-stained nuclei and examine the staining characteristics of stained p40 nuclei; 33,072 p40-stained nuclei were identified from these images. The p40 nuclei least squares means counts and staining characteristics are presented and contrasted in Table 4. Total p40positive nuclei counts in saline-infused quarters were greater than those in Staph. aureus-infused quarters (971 vs. $863 \pm 48$ nuclei; $P=0.036$ ); however, they did not differ between euthanasia days $(P=0.80)$, nor were counts affected by quarter treatment interacting with day of euthanasia $(P=0.99)$. Nuclear staining area of positively stained p40 nuclei was significantly lower in saline-infused quarters than in Staph. aureus-infused quarters (412 vs. $447 \pm 10$ pixels; $P=0.009$ ). The area of p40 nuclear staining was not different between mammary tissues collected 5 or $10 \mathrm{~d}$ postchallenge $(P$ $=0.18$ ). Eccentricity of p40-stained nuclei was not affected by the main effects of quarter treatment $(P=$ $0.24)$ or day of euthanasia $(P=0.94)$. No interaction was detected between quarter treatment and day of euthanasia in their effect on area or eccentricity of p40 nuclear staining $(P \geq 0.31)$.

\section{DISCUSSION}

\section{ESR1-Positive Nuclei Counts and Staining Characteristics}

A primary objective of this evaluation was to determine whether expression of ERS1 in mammary epithelial cell nuclei was altered by Staph. aureus IMI and the immune response initiated to address the IMI in glands that were simulated to rapidly grow and develop. There was a tendency for Staph. aureus-infused quarters to have fewer ESR1-positive nuclei than saline-infused quarters. This reduction in positive ESR1 nuclei is largely a function of reduced amounts of mammary epithelium in the Staph. aureus-infused quarters previously reported by Enger et al. (2018). In that report, we noted that the mammary tissues collected from these Staph. aureus-infused quarters contained 13\% less epithelium than saline-infused quarters. To adjust for this difference in epithelial area, the ESR1 nuclei counts for each Staph. aureus-infused quarter were multiplied by a $114.9 \%$ correction factor to normalize the nuclei counts to a comparable unit area of epithelium. When this correction was applied and the data were reanalyzed

Table 3. Least squares means counts and staining characteristics of progesterone receptor (PGR)-positive nuclei in saline- and Staphylococcus aureus-infused mammary gland tissues

\begin{tabular}{|c|c|c|c|c|c|c|c|c|}
\hline \multirow[b]{2}{*}{ Item } & \multicolumn{2}{|c|}{ Treatment } & \multirow[b]{2}{*}{ SEM } & \multirow[b]{2}{*}{$P$-value } & \multicolumn{2}{|c|}{ Euthanasia day } & \multirow[b]{2}{*}{ SEM } & \multirow[b]{2}{*}{$P$-value } \\
\hline & Saline & Challenge & & & d 5 & d 10 & & \\
\hline Nuclei, ${ }^{1}$ count & 520 & 440 & 45 & 0.02 & 492 & 468 & 60 & 0.78 \\
\hline Eccentricity & 0.666 & 0.658 & 0.006 & 0.19 & 0.672 & 0.652 & 0.007 & 0.06 \\
\hline Mean intensity, arbitrary units & 0.076 & 0.074 & 0.003 & 0.36 & 0.071 & 0.078 & 0.003 & 0.21 \\
\hline Integrated intensity, arbitrary units & 40.6 & 39.8 & 2.4 & 0.72 & 35.9 & 44.6 & 3.0 & 0.06 \\
\hline
\end{tabular}

${ }^{1}$ Data expressed as the number of positive cells observed in 8 parenchymal tissue fields of view.

${ }^{2}$ Mean nuclear area of PGR staining in positively stained nuclei. 
Table 4. Least squares means counts and staining characteristics of p40-positive nuclei in saline- and Staphylococcus aureus-infused mammary gland tissues

\begin{tabular}{|c|c|c|c|c|c|c|c|c|}
\hline \multirow[b]{2}{*}{ Item } & \multicolumn{2}{|c|}{ Treatment } & \multirow[b]{2}{*}{ SEM } & \multirow[b]{2}{*}{$P$-value } & \multicolumn{2}{|c|}{ Euthanasia day } & \multirow[b]{2}{*}{ SEM } & \multirow[b]{2}{*}{$P$-value } \\
\hline & Saline & Challenge & & & d 5 & d 10 & & \\
\hline Nuclei, ${ }^{1}$ count & 974 & 863 & 48 & 0.04 & 908 & 929 & 58 & 0.80 \\
\hline Eccentricity & 0.770 & 0.766 & 0.004 & 0.24 & 0.768 & 0.768 & 0.005 & 0.94 \\
\hline
\end{tabular}

${ }^{1}$ Data expressed as the number of positive cells observed in 8 parenchymal tissue fields of view.

${ }^{2}$ Mean nuclear area of p40 staining in positively stained nuclei.

using the exact same statistical model, the previously observed differences in the number of ESR1-positive nuclei in saline- and Staph. aureus-infused quarters abated (201 vs. $187 \pm 47$ nuclei, respectively; $P=$ 0.44). Accordingly, it is reasonable to expect that the percentage of positive ESR1 mammary epithelial cells does not differ between the saline- and Staph. aureusinfused quarters; however, it is recognized that Staph. aureus-infused quarters would contain a lower absolute number of ESR1-positive mammary epithelial cells than saline glands. Regardless, given that a difference in the percentage of positive nuclei was not indicated from this examination, it suggests that abundance of ESR1-positive nuclei is not contributing to reduced tissue areas of mammary epithelium in Staph. aureusinfused quarters (Enger et al., 2018).

Coinciding with the lack of differences in the number of ESR1-positive nuclei between treatments, there were similar staining characteristics of ESR1-positive nuclei in saline- and Staph. aureus-infused quarters. No differences in ESR1 nuclear staining area, eccentricity, or the staining intensity measures used were observed between treatments. The significance of ESR1 staining intensity has been highlighted in previous works, as Geiger et al. (2017) found that increased ESR1 staining intensity was associated with increased levels of mammary growth. Similarly, Tucker et al. (2016b) reported significant reductions in the mammary growth of calves treated with an antiestrogen, ablating the mammogenic effects of estradiol. This reduction in mammary growth was associated with substantial reductions in the staining intensity of ESR1-positive mammary epithelial cells. No differences were observed between ESR1 staining characteristics of saline- and Staph. aureus-infused quarters, indicating that IMI does not influence ESR1 abundance in mammary epithelial nuclei.

Fewer ESR1-positive nuclei were present in mammary tissues collected $5 \mathrm{~d}$ postchallenge than in those collected $10 \mathrm{~d}$ postchallenge. Two factors are expected to influence this result. First, all animals used in this experiment were synchronized to similar days of estrus before receiving estradiol and progesterone injections, confounding this observation with the effect of estrus day. It is well recognized and documented that expression of ovarian hormone receptors, specifically ESR1 in this instance, is temporal and can be influenced by reproductive status (Schams et al., 2003) and day of estrus (Spencer and Bazer, 1995; Silva et al., 2014). Second, because supraphysiologic dosages of estradiol and progesterone were administered to the used animals to stimulate rapid mammary growth, a reduction in the number of positive ESR1 nuclei was expected. Geiger et al. (2017) previously observed that continuous administration of estradiol to prepubertal heifers via an estradiol implant reduced the percentage of positive ESR1 mammary epithelial cells. Meyer et al. (2006) also observed a lower percentage of ESR1-positive mammary epithelial cells in heifers injected with estradiol once daily for $3 \mathrm{~d}$ before euthanasia compared with heifers not receiving exogenous estradiol. In essence, the fewer ESR1-positive nuclei in tissues collected $5 \mathrm{~d}$ postchallenge relative to those collected $10 \mathrm{~d}$ postchallenge could simply be an effect of time from the last estradiol injection. Should this result be examined in the future, it could be argued that synchronization of animals to similar days in estrus should be avoided so that this effect is not confounding; however, as a consequence, more interanimal variation would be expected. A primary goal of this study's design was to limit interanimal variation given this study's objective. The lack of differences between tissues collected 5 or $10 \mathrm{~d}$ postchallenge for the examined ESR1 staining characteristics indicates that the localization and abundance of ESR1 in positively stained epithelial nuclei appear to be consistent and not significantly influenced by day of euthanasia.

\section{PGR-Positive Nuclei Counts and Staining Characteristics}

Another key objective of this evaluation was to determine whether expression of PGR in mammary epithelial cell nuclei was affected by Staph. aureus IMI and the associated immune response. When the number of 
PGR-positive nuclei from Staph. aureus-infused quarters was adjusted in the exact same manner as done for the ESR1 nuclei counts (having $13 \%$ less epithelium than saline-infused quarters; Enger et al., 2018), no difference in the number of PGR-positive nuclei remained between saline- and Staph. aureus-infused quarters (520 vs. $505 \pm 49$ nuclei, respectively; $P=0.69$ ). This is similar to the observation that ESR1-positive nuclei counts were not influenced by quarter treatment but the Staph. aureus-infused quarters would still contain a lower absolute number of PGR-positive epithelial cells than uninfected glands. Scant literature exists describing the expression of PGR in bovine mammary glands with which to compare this observation, but it is expected that alterations in the number of PGR-positive mammary epithelial cells would affect the morphogenesis and branching of the glandular epithelial network. This is because PGR knockout in mice fails to develop side-branching alveolar structures in response to estrogen and progesterone treatment (Lydon et al., 2000). When mammary glands from these mice are transplanted into wild-type mice and pregnancy is established, a similar failure in lobular alveolar development occurs (Brisken et al., 1998). Regardless, given that the number of PGR-positive mammary epithelial cells did not differ when counts were adjusted for epithelial area, no influence from progesterone signaling is expected to contribute to changes in mammary morphogenesis.

The staining characteristic measures applied to positively stained PGR nuclei did not differ between salineand Staph. aureus-infused quarters. This result further substantiates the absence of PGR abundance affecting the reductions in mammary epithelial area and altering tissue morphology.

No difference in the number of PGR-positive nuclei was observed between mammary tissues collected 5 and $10 \mathrm{~d}$ postchallenge, suggesting that the number of PGRpositive nuclei is more constant and less temporal than the expression of ESR1 in mammary epithelial cells. On the other hand, differences were observed between euthanasia day in nuclear stained area, integrated intensity, and eccentricity. These staining characteristic measures are not entirely independent and are influenced by CellProfiler's functionality and the approach used to analyze the assigned images. Positively stained PGR nuclei were identified based on the presence of signal; because these images are grayscale, signal varied in intensity. For this analysis, a minimum intensity threshold was applied to the entire image set during the identification of positively stained nuclei. This was done to prevent misclassification of background signal and accurately identify positive nuclei. As a result, outlining of positive nuclei by CellProfiler was intensity dependent; therefore, if less intense staining was pres- ent inside a mammary epithelial cell nucleus, a smaller object would be outlined. Recognizing this effect, 2 intensity measures were used. Mean intensity depicts the mean signal intensity within a designated area, whereas integrated intensity is calculated by summing each pixel's intensity in the identified object. Thus, mean intensity is less likely to represent differences in receptor abundance given signal intensity was used as an identification criterion, whereas integrated intensity evaluates signal intensity in the context of a stained area. With this stipulation, the greater area of nuclear staining observed in mammary epithelial cell nuclei at $10 \mathrm{~d}$ postchallenge compared with $5 \mathrm{~d}$ postchallenge contributed to the observed differences in integrated intensities between the different euthanasia days. This indicates that the abundance of PGR receptor was greater in nuclei from tissues collected $10 \mathrm{~d}$ postchallenge. Again, the reasons for this increased expression are not entirely understood but are likely to be influenced by estrus day and time since last injection, as discussed previously.

\section{p40-Positive Nuclei Counts and Staining Characteristics}

In this preliminary evaluation, the effects of Staph. aureus IMI and the associated immune response were assessed on myoepithelial cells in nonlactating glands that were stimulated to grow rapidly. When the number of p40-positive nuclei was adjusted for Staph. aureusinfused quarters containing less epithelium, the earlierdescribed differences in number of positive nuclei in saline and Staph. aureus quarters abated (974 vs. 988 \pm 52 nuclei, respectively; $P=0.80)$. Aside from the number of mammary epithelial cells in the gland, the nuclear area of p40 staining was significantly greater in Staph. aureus-infused quarters. Reasons for this observation remain elusive but are presumed to be a result of myoepithelial cells responding to the presence of IMI and immune cell recruitment. Myoepithelial cells in the lactating gland are important for expressing milk from the alveolar lumen during milking, but these cells are also associated with gland morphogenesis and establishing epithelial cell polarity, as reviewed by Gudjonsson et al. (2005). In the bovine, myoepithelial cells in the glands of ovariectomized heifers are smaller and irregularly shaped relative to cells in glands from intact heifers (Safayi et al., 2012). Tucker et al. (2016a) also described altered myoepithelial cell organization in mammary glands from heifers treated with an antiestrogen and noted that myoepithelial cells were smaller and spaced farther apart relative to untreated heifers. Although the precise mechanisms responsible for the differential staining between saline- and Staph. 
aureus-infused quarters are unknown, some hypotheses can be made. First, given that myoepithelial cells are located basolaterally to mammary epithelial cells and help separate these secretory cells from the intralobular stroma compartment, we speculate that these cells may be affected by diapedesis of leukocytes across the epithelial cell layer. Additionally, myoepithelial cells are recognized to contribute to the synthesis and secretion of proteins that compose the basal membrane (Warburton et al., 1982) and may be making select proteins or synthesizing signaling factors in response to the localized inflammation. Last, it is unclear whether the observed increase in area of p40 nuclear staining reflects an increase in nuclear size or an increased abundance of p40. It is possible that nuclear reorganization, allowing for the differential expression of genes in response to the localized tissue inflammation, is contributing to the increased nuclear area of myoepithelial cells in Staph. aureus-infused quarters. Also, p40 has been previously discussed as having roles in directing the stratification of epithelium, and a greater abundance observed here may complement previously reported epithelial hyperplasia occurring in Staph. aureus-infected glands (Trinidad et al., 1990a). Given this observation, future investigations characterizing the effect of IMI on mammary tissues should also include examination of myoepithelial cells.

\section{CONCLUSIONS}

To our knowledge, this study is the first to investigate how IMI, and the ensuing immune response, affects the abundance and staining characteristics of ESR1 and PGR in mammary epithelial cells or how IMI affects myoepithelial cells in bovine mammary glands stimulated to rapidly grow. The results of this study indicate that Staph. aureus IMI has limited effects on the abundance of ESR1- and PGR-positive mammary epithelial cells or the staining characteristics of ESR1or PGR-positive mammary epithelial cells, signifying that the previously described mammary growth failures in Staph. aureus-infected glands are not resultant of changes in ESR1 or PGR expression. Myoepithelial cells were affected by Staph. aureus IMI as staining area of the utilized marker differed between saline- and Staph. aureus-infused glands, suggesting that these cells are involved with the gland response to IMI. The extent to which and action in which these cells are implicated in the gland response to IMI is unknown.

\section{ACKNOWLEDGMENTS}

This work was supported by a competitive USDA (Washington, DC) National Institute of Food and
Agriculture (NIFA) Agriculture and Food Research Initiative (AFRI) grant (2017-67011-26049) awarded to B. D. Enger, a Virginia Agricultural Council grant (VAC project no. 685) awarded to R. M. Akers, and professorship funds (Horace E. and Elizabeth F. Alphin Professorship) awarded to R. M. Akers.

\section{REFERENCES}

Akers, R. M. 1990. Lactation physiology: A ruminant animal perspective. Protoplasma 159:96-111.

Akers, R. M. 2017. A 100-year review: Mammary development and lactation. J. Dairy Sci. 100:10332-10352.

Andreotti, C. S., E. A. Pereyra, C. Baravalle, M. S. Renna, H. H. Ortega, L. F. Calvinho, and B. E. Dallard. 2014. Staphylococcus aureus chronic intramammary infection modifies protein expression of transforming growth factor beta (TGF-beta) subfamily components during active involution. Res. Vet. Sci. 96:5-14.

Arruda, A. G., S. Godden, P. Rapnicki, P. Gorden, L. Timms, S. S. Aly, T. W. Lehenbauer, and J. Champagne. 2013. Randomized noninferiority clinical trial evaluating 3 commercial dry cow mastitis preparations: I. Quarter-level outcomes. J. Dairy Sci. 96:4419-4435

Ball, S., K. Polson, J. Eminy, W. Eyestone, and R. M. Akers. 2000. Induced lactation in prepubertal Holstein heifers. J. Dairy Sci. 83:2459-2463.

Brisken, C., S. Park, T. Vass, J. P. Lydon, B. W. O'Malley, and R. A. Weinberg. 1998. A paracrine role for the epithelial progesterone receptor in mammary gland development. Proc. Natl. Acad. Sci. USA 95:5076-5081.

Capuco, A. V., and R. M. Akers. 1999. Mammary involution in dairy animals. J. Mammary Gland Biol. Neoplasia 4:137-144.

Capuco, A. V., R. M. Akers, and J. J. Smith. 1997. Mammary growth in Holstein cows during the dry period: Quantification of nucleic acids and histology. J. Dairy Sci. 80:477-487.

Capuco, A. V., S. Ellis, D. L. Wood, R. M. Akers, and W. Garrett. 2002. Postnatal mammary ductal growth: Three-dimensional imaging of cell proliferation, effects of estrogen treatment, and expression of steroid receptors in prepubertal calves. Tissue Cell 34:143-154.

Connor, E. E., M. J. Meyer, R. W. Li, M. E. Van Amburgh, Y. R. Boisclair, and A. V. Capuco. 2007. Regulation of gene expression in the bovine mammary gland by ovarian steroids. J. Dairy Sci. 90(Suppl. 1):E55-E65.

Croom, W. J., R. J. Collier, D. E. Bauman, and R. L. Hays. 1976 Cellular studies of mammary tissue from cows hormonally induced into lactation: Histology and ultrastructure. J. Dairy Sci. 59:12321246

Eberhart, R. J. 1986. Management of dry cows to reduce mastitis. J. Dairy Sci. 69:1721-1732.

Enger, B. D., C. E. Crutchfield, T. T. Yohe, K. M. Enger, S. C. Nickerson, C. L. M. Parsons, and R. M. Akers. 2018. Staphylococcus aureus intramammary challenge in non-lactating mammary glands stimulated to rapidly grow and develop with estradiol and progesterone. Vet. Res. 49:47.

Fox, L. K. 2009. Prevalence, incidence and risk factors of heifer mastitis. Vet. Microbiol. 134:82-88.

Geiger, A. J., C. L. M. Parsons, and R. M. Akers. 2017. Feeding an enhanced diet to Holstein heifers during the preweaning period alters steroid receptor expression and increases cellular proliferation. J. Dairy Sci. 100:8534-8543.

Gudjonsson, T., M. C. Adriance, M. D. Sternlicht, O. W. Petersen, and M. J. Bissell. 2005. Myoepithelial cells: Their origin and function in breast morphogenesis and neoplasia. J. Mammary Gland Biol. Neoplasia 10:261-272.

Howe, J. E., C. W. Heald, and T. L. Bibb. 1975. Histology of induced bovine lactogenesis. J. Dairy Sci. 58:853-860.

Kamentsky, L., T. R. Jones, A. Fraser, M. A. Bray, D. J. Logan, K. L. Madden, V. Ljosa, C. Rueden, K. W. Eliceiri, and A. E. 
Carpenter. 2011. Improved structure, function and compatibility for CellProfiler: Modular high-throughput image analysis software. Bioinformatics 27:1179-1180.

Knight, C. H., and M. Peaker. 1982. Development of the mammary gland. J. Reprod. Fertil. 65:521-536.

Long, E., A. V. Capuco, D. L. Wood, T. Sonstegard, G. Tomita, M. J. Paape, and X. Zhao. 2001. Escherichia coli induces apoptosis and proliferation of mammary cells. Cell Death Differ. 8:808-816.

Lydon, J. P., L. Sivaraman, and O. M. Conneely. 2000. A reappraisal of progesterone action in the mammary gland. J. Mammary Gland Biol. Neoplasia 5:325-338.

Lyons, W. R., C. H. Li, and R. E. Johnson. 1958. The hormonal control of mammary growth and lactation. Recent Prog. Horm. Res. $14: 219-248$.

Meyer, M. J., A. V. Capuco, Y. R. Boisclair, and M. E. Van Amburgh. 2006. Estrogen-dependent responses of the mammary fat pad in prepubertal dairy heifers. J. Endocrinol. 190:819-827.

Nickerson, S. C., C. W. Heald, T. L. Bibb, and M. L. McGilliard. 1978. Cytological effects of hormones and plasma on bovine mammary tissue in vitro. J. Endocrinol. 79:363-368.

Oliver, S. P., M. J. Lewis, B. E. Gillespie, H. H. Dowlen, E. C. Jaenicke, and R. K. Roberts. 2003. Prepartum antibiotic treatment of heifers: Milk production, milk quality and economic benefit. J. Dairy Sci. 86:1187-1193.

Owens, W. E., S. C. Nickerson, P. J. Washburn, and C. H. Ray. 1991. Efficacy of a cephapirin dry cow product for treatment of experimentally induced Staphylococcus aureus mastitis in heifers. J. Dairy Sci. 74:3376-3382.

Parsons, C. L. M., H. L. M. Tucker, R. M. Akers, and K. M. Daniels, 2018. Technical note: p40 antibody as a replacement for p63 antibody in bovine mammary immunohistochemistry. J. Dairy Sci. 101:7614-7617.

Safayi, S., N. Korn, A. Bertram, R. M. Akers, A. V. Capuco, S. L. Pratt, and S. Ellis. 2012. Myoepithelial cell differentiation markers in prepubertal bovine mammary gland: Effect of ovariectomy. J. Dairy Sci. 95:2965-2976.

Schams, D., S. Kohlenberg, W. Amselgruber, B. Berisha, M. W. Pfaffl, and F. Sinowatz. 2003. Expression and localisation of oestrogen and progesterone receptors in the bovine mammary gland during development, function and involution. J. Endocrinol. 177:305-317.
Silva, E. S., K. E. Scoggin, I. F. Canisso, M. H. Troedsson, E. L. Squires, and B. A. Ball. 2014. Expression of receptors for ovarian steroids and prostaglandin E2 in the endometrium and myometrium of mares during estrus, diestrus and early pregnancy. Anim. Reprod. Sci. 151:169-181.

Spencer, T. E., and F. W. Bazer. 1995. Temporal and spatial alterations in uterine estrogen receptor and progesterone receptor gene expression during the estrous cycle and early pregnancy in the ewe. Biol. Reprod. 53:1527-1543.

Sud, S. C., H. A. Tucker, and J. Meites. 1968. Estrogen-progesterone requirements for udder development in ovariectomized heifers. J. Dairy Sci. 51:210-214.

Trinidad, P., S. C. Nickerson, and R. W. Adkinson. 1990a. Histopathology of staphylococcal mastitis in unbred dairy heifers. J. Dairy Sci. 73:639-647.

Trinidad, P., S. C. Nickerson, and T. K. Alley. 1990b. Prevalence of intramammary infection and teat canal colonization in unbred and primigravid dairy heifers. J. Dairy Sci. 73:107-114.

Tucker, H. A. 1987. Quantitative estimates of mammary growth during various physiological states: A review. J. Dairy Sci. 70:1958-1966.

Tucker, H. A. 2000. Hormones, mammary growth, and lactation: A 41-year perspective. J. Dairy Sci. 83:874-884.

Tucker, H. L. M., K. L. Beaudry, C. L. M. Parsons, S. E. Ellis, and R M. Akers. 2016a. Impaired mammary development in tamoxifentreated prepubertal heifers is associated with altered development and morphology of myoepithelial cells. J. Dairy Sci. 99:1009310101.

Tucker, H. L. M., C. L. Parsons, S. Ellis, M. L. Rhoads, and R. M. Akers. 2016b. Tamoxifen impairs prepubertal mammary development and alters expression of estrogen receptor alpha (ESR1) and progesterone receptors (PGR). Domest. Anim. Endocrinol. 54:95-105.

Warburton, M. J., D. Mitchell, E. J. Ormerod, and P. Rudland. 1982. Distribution of myoepithelial cells and basement membrane proteins in the resting, pregnant, lactating, and involuting rat mammary gland. J. Histochem. Cytochem. 30:667-676.

Woodward, T. L., W. E. Beal, and R. M. Akers. 1993. Cell interactions in initiation of mammary epithelial proliferation by oestradiol and progesterone in prepubertal heifers. J. Endocrinol. 136:149-157. 